Article

\title{
Exploring the Impact of Terminators on Transgene Expression in Chlamydomonas reinhardtii with a Synthetic Biology Approach
}

\author{
Katrin Geisler $^{1}{ }^{\circledR}$, Mark A. Scaife ${ }^{1,2}{ }^{,}$Paweł M. Mordaka ${ }^{1}$, Andre Holzer ${ }^{1}{ }^{\circledR}$, Eleanor V. Tomsett ${ }^{1}$, \\ Payam Mehrshahi ${ }^{1}$, Gonzalo I. Mendoza Ochoa ${ }^{1}$ (i) and Alison G. Smith ${ }^{1, *}$ \\ 1 Department of Plant Sciences, University of Cambridge, Downing Street, Cambridge CB2 3EA, UK; \\ kg404@cam.ac.uk (K.G.); drmscaife@gmail.com (M.A.S.); pmm63@cam.ac.uk (P.M.M.); \\ ah830@cam.ac.uk (A.H.); eleanor.tomsett@outlook.com (E.V.T.); pm579@cam.ac.uk (P.M.); \\ gim23@cam.ac.uk (G.I.M.O.) \\ 2 Mara Renewables Corporation, Dartmouth, NS B2Y 4T6, Canada \\ * Correspondence: as25@cam.ac.uk; Tel.: +44-1223-333952
}

Citation: Geisler, K.; Scaife, M.A.; Mordaka, P.M.; Holzer, A.; Tomsett, E.V.; Mehrshahi, P.; Mendoza Ochoa, G.I.; Smith, A.G. Exploring the Impact of Terminators on Transgene Expression in Chlamydomonas reinhardtii with a Synthetic Biology Approach. Life 2021, 11, 964 . https: / / doi.org/10.3390/life11090964

Academic Editors: Paul F. South, Pasquale Stano and Stéphane D. Lemaire

Received: 31 July 2021

Accepted: 9 September 2021

Published: 14 September 2021

Publisher's Note: MDPI stays neutral with regard to jurisdictional claims in published maps and institutional affiliations.

Copyright: (c) 2021 by the authors. Licensee MDPI, Basel, Switzerland. This article is an open access article distributed under the terms and conditions of the Creative Commons Attribution (CC BY) license (https:// creativecommons.org/licenses/by/ $4.0 /)$.

\begin{abstract}
Chlamydomonas reinhardtii has many attractive features for use as a model organism for both fundamental studies and as a biotechnological platform. Nonetheless, despite the many molecular tools and resources that have been developed, there are challenges for its successful engineering, in particular to obtain reproducible and high levels of transgene expression. Here we describe a synthetic biology approach to screen several hundred independent transformants using standardised parts to explore different parameters that might affect transgene expression. We focused on terminators and, using a standardised workflow and quantitative outputs, tested 9 different elements representing three different size classes of native terminators to determine their ability to support high level expression of a GFP reporter gene. We found that the optimal size reflected the median size of element found in the C. reinhardtii genome. The behaviour of the terminator parts was similar with different promoters, in different host strains and with different transgenes. This approach is applicable to the systematic testing of other genetic elements, facilitating comparison to determine optimal transgene design.
\end{abstract}

Keywords: algae; heterologous protein expression; modular assembly; quantification of transgene expression; standardized workflow; terminator; $3^{\prime}$ UTR

\section{Introduction}

Of the plethora of microalgae identified to date, only a small number have been studied extensively and even fewer have been developed sufficiently for their laboratory culture, biochemical analysis, and genetic manipulation to be considered routine [1]. Of these, the green alga Chlamydomonas reinhardtii (Chlorophyta) is the best studied, and over the course of $>30$ years of basic and applied research, its study has contributed to our understanding of many fundamental biological processes, in particular photosynthesis [2] and motility [3]. It is often referred to as a model alga, and this designation has helped to galvanise efforts to develop several essential resources, which are now enabling the use of C. reinhardtii for biotechnology [4-6]. These include well-established basic laboratory protocols for culturing and molecular analysis, excellent genetics [7], fully sequenced genomes for the nucleus, mitochondrion and chloroplast [8], and efficient transformation protocols for all three genomes [9]. Routinely this is via electroporation [10], but simple vortexing with glass beads is also possible [11]. Such resources have led directly to the generation of an impressive array of sequences to enable transgene expression, including selectable markers, reporter genes [12-14] and promoter elements (reviewed in [4,5]), as well as gene silencing techniques that utilise RNA interference (RNAi) [15] or artificial microRNAs (amiRNAs) [16] and gene editing via CRISPR/Cpf1 [17]. In parallel, the implementation of synthetic biology approaches, most notably the adoption of standard 
parts and modular cloning methods, has enabled much more rapid generation of constructs for transformation and effective comparison between different designs, culminating in the generation of a kit of over 100 standardized elements [18], available at the Chlamydomonas Resource Center [19].

As a result, there have been many reports of the use of $C$. reinhardtii as a host for the production of high-value compounds and of individual proteins, particularly those with therapeutic potential (reviewed in $[4,20,21]$ ). The chloroplast has proved a versatile expression platform for recombinant proteins since it naturally accumulates high levels of soluble protein. C. reinhardtii is one of only two organisms, the other being Nicotiana spp., where chloroplast transformation is robust and routine. Transgenes can be introduced into the chloroplast genome by homologous recombination at precise locations using flanking homology arms bordering the gene(s) to be inserted. For several proteins, yields of up to $5 \%$ total soluble protein have been reported (reviewed in [22]). Disulphide bond formation occurs readily, but there is no glycosylation system in the organelle, so it is not suitable for expression of all proteins. In fact, more common is the introduction of transgenes into the nucleus, allowing a greater range of genes to be expressed, and the protein products can then be targeted to the appropriate subcellular location, including the chloroplast, by inclusion of appropriate targeting sequences in the genetic constructs [23]. Many of these are now available via the Chlamydomonas Spatial Interactome [24], which provides the means to target proteins not just to the organelles themselves, but also to specific sub-compartments within them [25]. As well as single proteins [26], there is increasing success in expressing metabolic enzymes to allow production of compounds such as the diterpenoids casbene, taxadiene, and 13R-(+)-manoyl oxide [27].

However, whilst it is straightforward to obtain large numbers of nuclear transformants of C. reinhardtii, transgenes are inserted essentially randomly. Ligation of trans-DNA is known to occur at sites of double-stranded breaks by the non-homologous end joining repair pathway. Insertion of the transgene is often preceded by endonucleolytic cleavage of DNA (with preference at CA/TG consensus motif), which can result in the unexpected fragmentation and/or rearrangement of the transgene [28]. As a consequence, large numbers of transformants must be screened to ensure they contain the appropriate construct. More significantly, the level of transgene expression is often very low, with many transformants not expressing above background levels. Worse still, it is subject to gene silencing over time, most likely at the transcriptional level [29], particularly if selection is not maintained. Although the reasons for this extensive silencing are not clear, several empirical approaches have been followed to try to overcome low or unreliable transgene expression (reviewed in [30]).

A particular milestone in the field of C. reinhardtii engineering was achieved as the result of UV mutagenesis of a strain that was only weakly expressing the CRY1 gene that confers resistance to the alkaloid emetine [31]. Two mutants, UVM4 and UVM11, were found that were resistant to much higher concentrations of emetine, and they were subsequently shown to express another, newly transformed, heterologous transgene at high frequency and to high levels. The common mutation between these two lines was subsequently shown to be a defect in the SIR2 gene encoding a histone deacetylase [32].

As well as the genetic background of the host strain, design of constructs is also crucial. C. reinhardtii has very high GC content (with an average of $64 \%$ throughout the nuclear genome and 68\% in coding sequences [8]), and a strict biased codon usage [33]. Efficient transgene expression is achieved only by using appropriate codon usage, which in practice means that heterologous genes have to be synthesised. Inclusion of introns, which facilitate interaction of the RNA polymerase with the spliceosome, is similarly necessary, with an increase in expression of up to 5-fold observed over transgenes without introns in several studies (e.g., [34,35]). Finally, there have been extensive studies on optimal regulatory elements, particularly promoters. For example, there are several strong promoters that have been widely used including for the small subunit of Rubisco (RBCS) and a Photosystem I subunit (PSAD), but these are both preferentially expressed in the light [36] and C. reinhardtii does not appear to have strong constitutive promoters expressed 
under all conditions. Instead, researchers have developed novel promoters, such as the chimeric HSP70A/RBCS2 $i$ promoter [37] and several completely synthetic promoters based on combining sequence and nucleotypic characteristics of known promoters [38]. The choice of terminator has also been found to play a role. Kumar et al. [39] tested three different terminators in combination with seven different promoters. They found that maximal expression of the luciferase reporter gene was obtained using the PSAD promoter together with the PSAD terminator, although a $\beta 2$-tubulin promoter/PSAD terminator combination was also effective, indicating that it was not simply cognate pairing that provided optimal expression levels. A further study identified terminators of highly expressed genes for a ribosomal subunit (RPL23) and ferredoxin (FDX1) as supporting high levels of luciferase reporter activity [40], and that the former was more effective than the PSAD terminator in expressing the sh-ble gene conferring zeocin-resistance.

We decided to explore further how terminators might impact transgene stability in C. reinhardtii. Our best understanding of the role(s) of terminators comes from studies in mammals and yeast, but similar features are known to operate in plants [41]. Terminators and their interaction with the polyadenylation complex are crucial for appropriate transcriptional termination and $3^{\prime}$ end formation [42], including addition of the poly(A) sequence, which is essential for stabilisation of the mRNA, export to the cytoplasm and efficient translation. In addition, it has been proposed that terminators can stimulate initiation of transcription, where interaction between the $5^{\prime}$ and $3^{\prime}$ ends of the gene-through so called gene loops-facilitate recycling of RNA polymerase II from the end of the gene to the start $[43,44]$. Detailed dissection of plant terminator sequences has revealed that in addition to a polyadenylation motif of AAUAAA or AAUAAA-like, which is 10-20 nt from the cleavage site, there are several conserved near- and far-upstream elements (NUEs and FUEs respectively). The importance of these elements was confirmed by a study in maize where several ubiquitin terminators were analysed for their ability to drive reporter gene expression [45]. In contrast, a genome-wide analysis of the poly(A) sites in C. reinhardtii indicated that the conserved UGUAA motif (which is itself distinct from that in plants and animals) is the major cis-element involved in polyadenylation, with no other conserved sequences identified [46].

Here we describe an analysis of the size ranges of terminators in the C. reinhardtii genome and then test nine different terminators that represent the different size classes for their efficacy in regulation of transgene expression in the C. reinhardtii nucleus. We also present a standard workflow that has been developed in our laboratory for the effective nuclear transformation of $C$. reinhardtii and the quantitative measurement of several parameters to facilitate optimisation of construct design.

\section{Materials and Methods}

\subsection{C. reinhardtii Strain Information}

Three different C. reinhardtii strains were used in this work: the cell wall deficient strains CC-1615 cw15 mt- (cw15) (Chlamydomonas Resource Centre, City, State Abbrev if USA, Country) and UVM4 [31], and the wild-type strain 12 (WT12), which is a walled strain derived from strain CC-124 137c (mt-nit1 nit2) and was obtained from Saul Purton (University College London, London, UK) [47]. C. reinhardtii cultures were grown in liquid or solid Tris-acetate phosphate (TAP) medium with Kropat's trace elements [48], but excluding selenium at $25{ }^{\circ} \mathrm{C}$ under continuous light conditions (light intensity of 80-100 $\left.\mu \mathrm{E} \cdot \mathrm{m}^{-2} \cdot \mathrm{s}^{-1}\right)$.

\subsection{Bioinformatic Analysis}

Version 5.6 of the Chlamydomonas reinhardtii (CC-503 v5.6) reference genome assembly [8] was downloaded from JGI's plant genomics research database Phytozome 12 (https://phytozome.jgi.doe.gov/pz/portal.html) in March 2019. Filtered gene models 'Creinhardtii_281_v5.6.gene.gff3' were employed, together with custom R scripts, to determine genomic distance between subsequent genes and other genomic features, respectively. 
Annotations included coding regions of genes as well as position of individual $5^{\prime}$ and $3^{\prime}$ UTRs. Histograms were plotted applying a binsize of $100 \mathrm{bp}$.

Gene expression ranking was performed based on Fragments Per Kilobase of transcript per Million (FPKM) mapped reads values of 17,824 nuclear, chloroplast or mitochondrial encoded genes obtained by RNA-seq of $C$. reinhardtii over a $24 \mathrm{~h}$ period [36]. FPKM values from Strenkert et al. [36] were used to calculate mean expression levels, standard deviation as well as expression ranking. For expression over the diurnal cycle FPKM values from all timepoints were used.

\subsection{Plasmid Design and Assembly}

Constructs were made using parts encoding promoters, $5^{\prime}$ UTRs and terminators from C. reinhardtii genomic DNA as well as in-house plasmid templates for reporter genes and selectable markers generated by the Smith laboratory (University of Cambridge, Cambridge, UK) or colleagues in other groups, as described in Tables S2 and S3. Genetic parts were amplified using primers listed in Table S1 and PCR products were purified with the Monarch PCR \& DNA Clean up kit (NEB, Hitchin, UK). Parts were assembled into plasmid constructs mainly by Gibson assembly [49] using the isothermal method, at $50^{\circ} \mathrm{C}$ for $1 \mathrm{~h}$. Alternatively, constructs were generated following standard Golden Gate (GG) cloning according to the modular cloning (MoClo) system [50,51], using parts from the Chlamydomonas MoClo toolkit [18] and parts that were created for this work. If necessary, parts were domesticated removing BpiI and BsaI sites by PCR-based mutagenesis and cloned into corresponding vectors by GG cloning using either BsaI (NEB) or BpiI (Thermo Fisher Scientific, UK) depending on the level of the destination MoClo vectors, together with T4 Ligase (NEB). Plasmids were propagated in E. coli (NEB 5-alpha competent cells), isolated using the Monarch Plasmid preparation kit (NEB) and verified by sequencing (Source Bioscience, Cambridge, UK; Genewiz, Bishop's Stortford, UK) or restriction digestion.

\subsection{Chlamydomonas Transformation and Culturing}

Plasmids were linearised and transformed into C. reinhardtii via electroporation as previously described [52]. Plasmids generated by Gibson assembly were linearised with ScaI or if the METE terminator was present with NcoI, resulting in flanking DNA sequence of $881 \mathrm{bp}$ and $765 \mathrm{bp}$ respectively. Plasmids generated via GG cloning were linearised with EcoRV resulting in a flanking region of $1088 \mathrm{bp}$. The impact of this change to the flanking DNA sequence and length was not tested in this investigation. After checking for complete digestion by gel electrophoresis, $1 \mu \mathrm{g}$ of plasmid DNA was used per transformation without any further purification steps. Primary selection was performed on TAP agar plates supplemented with zeocin $\left(10 \mu \mathrm{gL}^{-1}\right)$ or paromomycin $\left(10 \mathrm{mg} \mathrm{L}^{-1}\right)$. Single colonies (primary transformants) were transferred to 96 well microtitre plates for culture in $200 \mu \mathrm{L}$ TAP liquid media, supplemented with antibiotics $\left(5 \mathrm{mg} \mathrm{L}^{-1}\right.$ zeocin or $10 \mathrm{mg} \mathrm{L}^{-1}$ paromomycin). The microtitre plates, sealed with micropore tape, were incubated under constant light $\left(120 \mu \mathrm{mol}\right.$ photon $\left.\mathrm{m}^{-2} \mathrm{~s}^{-1}\right)$ at $25^{\circ} \mathrm{C}$. Before subculturing cells were resuspended by pipetting up and down to avoid any cell clumping and $10 \mu \mathrm{L}$ of culture was transferred to $200 \mu \mathrm{L}$ of fresh media. Following three sequential subcultures, each lasting seven days, those cell lines that remained viable were dubbed stable transformants and assessed for the expression of GFP via confocal microscopy (TCS SP5, Leica Microsystems, Wetzlar, Germany). For analysis of the different terminators, promoters and host strains, each construct was tested in at least three independent transformations and the results presented are the average of $>288$ transformed lines.

\subsection{Confocal Laser Scanning Microscopy}

C. reinhardtii transformants carrying the Ble-GFP expression cassettes were grown for seven days in TAP media before visualization in a confocal laser scanning microscope (TCS SP5, Leica Microsystems, Wetzlar, Germany). For most of the experiments, pictures were taken for more than 30 individual transformed cell lines per construct. The exact 
number dependent on the survival under zeocin selection, therefore we present the data as percentage of the viable cell lines. For the comparison of no selection versus selection workflow (Figure S1) all viable cell lines from 24 initially selected transformants were investigated. For each cell line two to five pictures were taken for each sample using the $40 \times 1.25$ oil corrected objective. For screening purposes, a zoom of 1 was used and pictures were taken with a line average of 1 . The number of cells in these overview pictures (resolution 8 bits, $512 \times 512$ pixels) was dependent on the cell density of each cell line and varied from 3 to 80 cells. Throughout a single experiment the settings were kept the same. In general, a pinhole size of $68 \mu \mathrm{m}$ was used. Images were acquired from a white light source with excitation at $476 \mathrm{~nm}$ and emission detection between 485 and $518 \mathrm{~nm}$ for the GFP channel (Gain 700-825 V, Offset 0\%), and between 650 and $720 \mathrm{~nm}$ for the chlorophyll channel (Gain 550-600 V, Offset 0\%).

\subsection{Statistical Tests}

Transformation efficiencies were recorded as number of colonies appearing on selection plates after 7-10 days of growth for strains cw15 and UVM4, and 15-20 days for WT12 transformants. Algal growth in microtitre plates and GFP expression was assessed and scored after 7 days of growth. Values are given as means of at least three independent experiments \pm standard error of the mean. Algal lines transformed with the RBCS2 terminator construct were used for comparison unless otherwise stated and two-tailed Mann-Whitney U-tests were performed to determine statistical significance. A $p$-value of $<0.05$ was considered statistically significant.

\section{Results}

\subsection{Transgene Instability: A Recurrent Problem in C. reinhardtii}

Transformation of a cell wall-deficient strain of $C$. reinhardtii such as cw15 by electroporation using an optimised protocol [10] will routinely yield $>1000$ transformants per $\mu \mathrm{g}$ of plasmid DNA within 7-10 days of plating on selective medium. However, only a proportion of these colonies will express the transgene effectively. We demonstrate this with results from a typical experiment illustrated in Figure 1. C. reinhardtii cw15 cells were transformed with a construct encoding a Ble-GFP fusion protein under the control of the HSP70A/RBCS2 chimeric promoter (AR promoter; [37]) and RBCS2 $3^{\prime} \mathrm{UTR} /$ terminator, a combination that has been widely used in the literature [18]. The sh-ble gene from Streptoalloteichus hindustanus encodes a zeocin-binding protein, which is targeted to the nucleus, so that the green fluorescent protein (GFP) is also localises there [12]. We included the intron 1 of $R B C S 2$ (RBCS2i1) in the coding region of the Ble-GFP fusion protein, which has been shown to increase transgene expression in $C$. reinhardtii $[35,53]$. Transformants were selected on TAP agar plates containing $10 \mu \mathrm{g} / \mathrm{mL}$ zeocin and then 96 colonies were picked into liquid medium (TAP $+5 \mu \mathrm{g} / \mathrm{mL}$ zeocin) in a microtitre plate and subcultured every seven days. Cell growth was scored and recorded before every subculture. After three subcultures many of the initially picked lines were no longer able to grow under zeocin selection pressure (Figure 1, step 3). Those that were still viable were screened via confocal microscopy to identify lines that were expressing GFP (step 4). Some lines (e.g., line A3) showed high levels of fluorescence, whilst others (such as C12) had low level expression. These were both scored as expressing above the threshold. Approximately 80\% (e.g., line D4) did not express GFP above the detection limit. Thus, even when using a fusion protein, there was still variable expression of the GFP reporter in those that expressed sufficient Ble to be zeocin-resistant.

We also compared viability in zeocin in a selective versus a no selection workflow (Figure S1). When initially picked, transformants were subcultured in TAP without zeocin and 95 out of 96 strains survived after three subcultures. Subsequently, these strains were subjected to two rounds of subculturing in TAP with zeocin and only $49 \%$ of lines were still viable, comparable to the $41 \%$ that were cultured under constant selection pressure. Analysing a subset of these strains, we recorded a similar number of transformants showing 
threshold GFP expression across both workflows. As a similar number of GFP-expression strains were identified and considering the substantial loss of viable cell lines when testing for zeocin resistance after initial subculturing in non-selective media, we decided to use selection immediately in all subsequent experiments, since otherwise this extended the experiment by $2-3$ weeks.

\section{Typical experimental workflow}

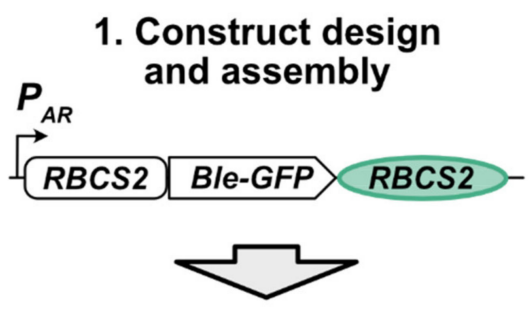

\section{Chlamydomonas transformation and selection}

Selection plates

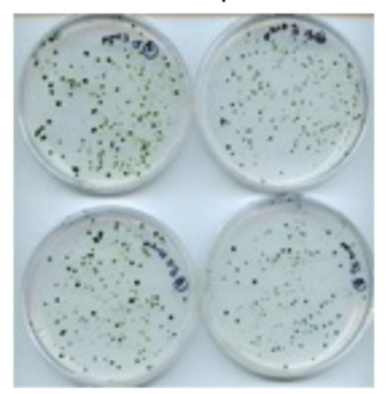

3. Colonies transferred to 96-well plates. Subcultured every 7 days

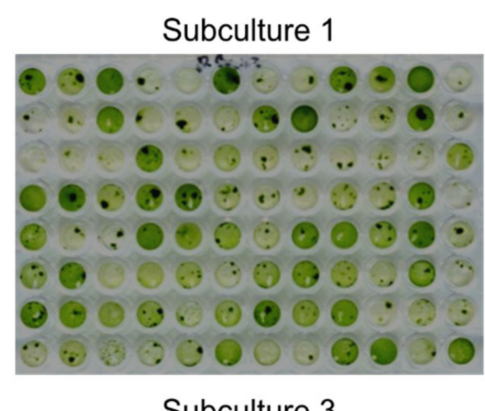

Subculture 3

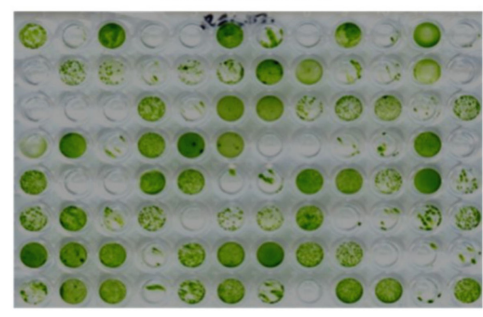

\section{Viable cell lines screened for GFP signal}

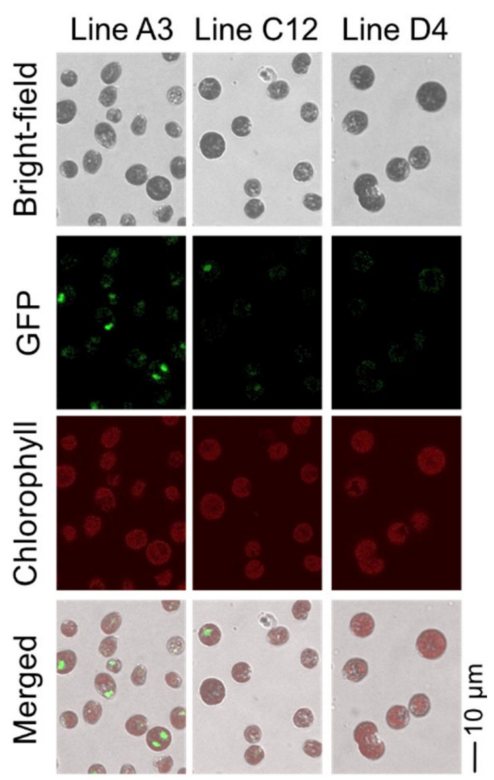

Figure 1. Typical experiment workflow of C. reinhardtii transformation and selection of stable transgenic lines. (Step 1): Schematic of a DNA construct expressing a target transgene. A Ble-GFP reporter CDS is under the control of the $A R$ promoter, and the RBCS2 terminator. (Step 2): After transformation of $C$. reinhardtii strain cw15 with a DNA construct, transformants are selected on agar plates containing zeocin. (Step 3): Initially obtained colonies are transferred to TAP liquid media in microtitre plates, supplemented with zeocin, in a 96-well microtiter plate and grown in constant light, subculturing every seven days. The growth of the different cell lines is recorded. (Step 4): After 3 subcultures, viable cell lines (ie zeocin-resistant) are screened for GFP expression via confocal microscopy. Representative images for three different cell lines (named according to the position in the 96-well plate) are shown using the brightfield, GFP and chlorophyll channels, as well as a merged figure of all three channels.

\subsection{Influence of Terminators on Transgene Expression in C. reinhardtii}

The systematic workflow we developed offered the means to screen large numbers of transformants to allow quantitative comparison between different constructs. We decided to explore in detail the impact of terminator choice on stability and level of gene expression since this has received less attention than other aspects of construct design [30]. Previous observations that using native features of the C. reinhardtii genome such as codon usage [33] and inclusion of introns $[35,54]$ had a major impact on transgene expression, led us to reason that the same would be true for terminators. Accordingly, data from the $C$. reinhardtii genome (CC-503 v5.6) [8] were employed to assess the range and abundance of annotated features of all protein-coding genes, including the coding sequence and $5^{\prime}$ UTRs (Figure S2) and $3^{\prime}$ UTRs (Figure 2a). The annotated $3^{\prime}$ UTRs were delineated using transcriptome data sets [8] and include the canonical C. reinhardtii polyadenylation sequence of UGUAA $[46,55$ ] and so are a proxy for the terminator sequence. The size distribution graph shows that a considerable number of genes $(\sim 7,407$; green bars in Figure $2 a)$ have $3^{\prime}$ UTRs of between 100-600 bp. Around 11,000 genes have a predicted $3^{\prime}$ UTR of $>600$ bp (blue bars), and a 
smaller proportion ( 686 genes; orange bars) have short $3^{\prime}$ UTRs of $<100 \mathrm{bp}$. Across the whole genome the median $3^{\prime}$ UTR length is $681 \mathrm{bp}$ and the median distance between the $3^{\prime}$ UTR of one gene and $5^{\prime}$ UTR of the subsequent gene is 3,059 bp (Figure S2).

Table 1. Candidate terminators tested in C. reinhardtii. The first three (RPL31, RPS29, RPL11) are short terminators (<100 bp), RBCS2, PSAD and THI4 are classified as medium sized (100-600 bp), and METE, CA1, NIT1 are long terminators ( $>600 \mathrm{bp})$. The predicted length based on the use of gene model analysis as well as the length of DNA parts used in this study is shown in base pairs (bp). The expression rank over the diurnal cycle was determined by comparing the mean FPKM values over all time points (data from [36]). For further details see Supplementary Table S4.

\begin{tabular}{cccccc}
\hline Gene ID & \multirow{2}{*}{ Name } & Annotation & $\begin{array}{c}\text { Length (bp) Gene } \\
\text { Model }\end{array}$ & $\begin{array}{c}\text { Length (bp) } \\
\text { Used }\end{array}$ & Expression Rank \\
\hline Cre12.g489153 & RPL31 & Ribosomal protein L31 & 34 & 34 & 42 \\
Cre08.g358556 & RPS29 & Ribosomal protein S29 & 59 & 58 & 79 \\
Cre01.g027000 & RPL11 & Ribosomal protein L11 & 60 & 60 & 63 \\
Cre02.g120150 & RBCS2 & Rubisco small subunit 2 & 179 & $221^{1}$ & 12 \\
Cre05.g238332 & PSAD & Photosystem I subunit D & 357 & $336^{2}$ & 80 \\
Cre04.g214150 & THI4 & Thiamine thiazole synthase & 481 & 481 & 734 \\
Cre03.g180750 & METE & Cobalamin-independent & 733 & 733 & 345 \\
Cre04.g223100 & CA1 & Carbonionine synthase & 786 & 786 & 300 \\
Cre09.g410950 & NIT1 & Nitrate reductase 1 & 888 & 888 & 15537 \\
\hline
\end{tabular}

${ }^{1}$ Same part used as in [39] ${ }^{2}$ Shortened, still includes UGUAA Poly(A) motif and a further $33 \mathrm{bp}$.

(a)

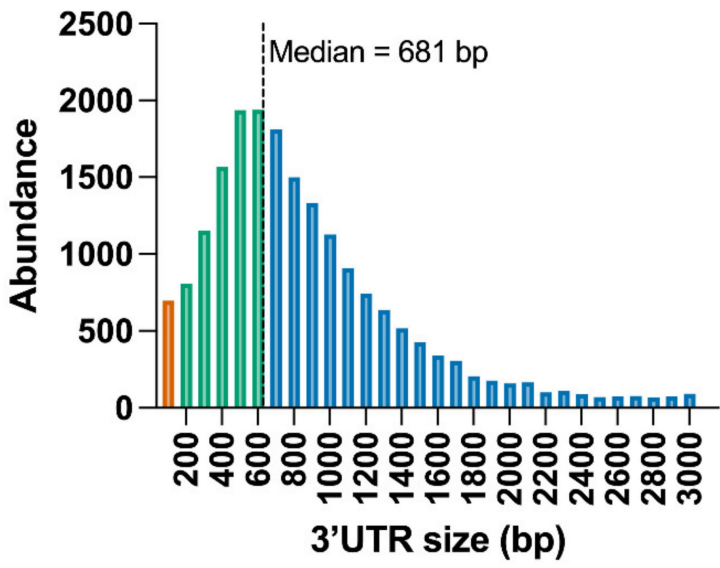

(b) Parts used to test terminators

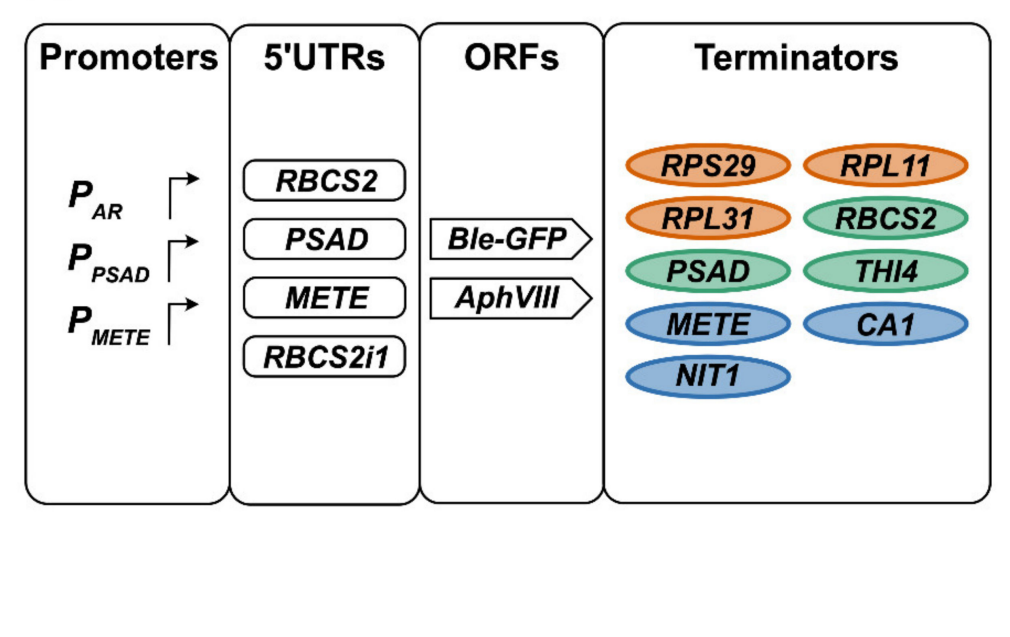

Figure 2. Analysis of $3^{\prime}$ UTR size and construct design to study terminators in C. reinhardtii. (a) Distribution of annotated $3^{\prime}$ UTR sizes from the $C$. reinhardtii genome v5.6 (b) Schematic of different promoter, $5^{\prime}$ UTR, ORF and terminator parts used throughout this work. Orange represents $3^{\prime}$ UTR/terminators of $<100$ basepairs (bp), green represents medium length $3^{\prime}$ UTRs (100-600 bp) and blue indicates long $3^{\prime}$ UTRs with a length $>$ than the median. For details see Table 1 . Promoter $P_{A R}$ is a $H S P 70 / R B C S 2$ promoter fusion.

We took examples of these three different size classes of terminators to use as an independent variable in transgene expression using the workflow described above, testing their influence on transgene expression and stability. Three short elements chosen were ribosomal proteins, RPS29, RPL11, and RPL31, that were all under $100 \mathrm{bp}$. For those of medium length (150-600 bp), two (RBCS2 and PSAD) have been widely used in C. reinhardtii constructs, whilst the third, THI4, encoding an enzyme of thiamine biosynthesis, is novel. The three representatives of the longer terminators with a length above $700 \mathrm{bp}$, were METE (encoding cobalamin-independent methionine synthase; [56]), NIT1 (encoding nitrate reductase) and a carbonic anhydrase gene, $C A 1$ (Figure 2b, Table 1). All terminators used 
in this study carried the UGUAA poly(A) motif. We also included a no-3' UTR control that had no element beyond the stop codon.

In choosing these terminator elements, we considered their expression ranking by analysing previously published RNA-seq data of C. reinhardtii over a $24 \mathrm{~h}$ period [36]. While there are diurnal expression differences in nearly every gene, the terminators chosen were from genes ranked highly (above rank 350) averaged over the entire diurnal cycle (Table 1, Table S4); terminators from highly expressed genes had previously shown to be effective in transgene expression [40]. The only exception is the NIT1 terminator, whose average expression was ranked 15,537 in the analysed data set, but which is highly expressed in nitrogen deplete conditions [57].

Although more than half of all nuclear gene transcripts in C. reinhardtii have a $3^{\prime}$ UTR longer than $1000 \mathrm{bp}(58 \%)$, these are underrepresented (11/100) among the top constitutively expressed nuclear genes, which in contrast are strongly associated with short $3^{\prime}$ UTRs (Dataset S2). Moreover, since our aim was to identify terminators that would be useful as standard synthetic biology parts for construct assembly, we choose not to use terminators longer than $1000 \mathrm{bp}$ since this might interfere with their assembly via modular cloning methods. It should also be noted that in previous studies [39] a 548 bp version of the PSAD terminator was used. As the annotated gene model predicted a shorter terminator, we opted to test a shorter version of $336 \mathrm{bp}$, which includes the UGUAA poly(A) signal, again to minimise the size of the terminator for cloning.

In an initial experiment we assembled constructs in which Ble-GFP, containing the intron RBCS2i1, was under the control of the $A R$ promoter and the RBCS2 $5^{\prime} \mathrm{UTR}$, with each of the selected terminator candidates (Figure $2 b$ ). After plasmid linearisation, the different constructs were transformed into $C$. reinhardtii $\mathrm{cw} 15$ and transformants were selected on TAP plates containing zeocin. The transformation efficiency differed considerably between them. With the RBCS2 terminator (Figure 1) as a base line, we obtained $\sim 2.47 \times 10^{3}$ colonies per $\mu \mathrm{g}$ DNA (Figure $3 \mathrm{a}$ ). This is not statistically different from the number of colonies obtained when a no- $3^{\prime}$ UTR construct was used $\left(\sim 1.63 \times 10^{3}\right.$ colonies per $\mu$ g DNA). In contrast, the construct with the $C A 1$ terminator produced $5.50 \times 10^{3}$ colonies per $\mu \mathrm{g}$ DNA, a 2.2-fold increase over the base case that was statistically significant $(p<0.01)$. The PSAD, THI4, METE and NIT1 terminators also increased the number of zeocin resistant transformants, but these were not statistically significant. None of the shorter terminators improved the transformation efficiency, despite being ranked in the top 100 most highly expressed genes in the diurnal RNA-seq dataset (Table $1 ;$ [36]). The trend suggests that the longer the terminator, the higher the number of initially selected transformants.

We next investigated the stability of primary transformants through multiple rounds of subculturing under antibiotic selection pressure as described above (Figure 1). Figure $3 b$ shows that the number of zeocin resistant cell lines decreased for all libraries over the course of this analysis. For our baseline construct carrying the RBCS2 terminator, in three independent experiments an average of $74 \%$ of the initial lines were zeocin resistant after three subcultures (Figure 3c). The rate of decline in viability can be seen in the time course of Figure 3b, with the most rapid being in the no-3' UTR cell lines, which had fewer than $50 \%$ surviving under constant selection pressure at subculture 3 (Figure 3c). All other tested terminators showed improved transgene stability, measured as zeocin-resistance, compared to the no- $3^{\prime}$ UTR library. CA1 demonstrated the greatest level of stability with a reduction in viability over the experimental period of just $13 \%$. For the remaining seven terminators, a reduction in viability of between $20-32 \%$ was observed.

The stable cell lines were then examined via confocal microscopy to determine which were expressing detectable levels of GFP. As shown in Figure 3d, 23\% of the zeocin resistant cell lines carrying the RBCS2 terminator accumulated GFP above the threshold level of detection (Figure 3d), comparable to that reported in other studies [33]. Similarly low percentages of GFP positive cell lines were observed for strains transformed with the short terminators of RPS29, RPL11 and RPL31 (15-35\% GFP positive) or the no-3' UTR construct (25\% GFP positive). The best performing constructs contained terminators from PSAD, 
with $83 \%$ GFP positives, and CA1, with $81 \%$ GFP positives, performing better than the RBCS2 terminator $(p<0.01)$. The THI4, METE and NIT1 terminators resulted in $60-70 \%$ GFP positives (Figure 3d), also significantly better than the shorter elements $(p<0.05)$.

(a)

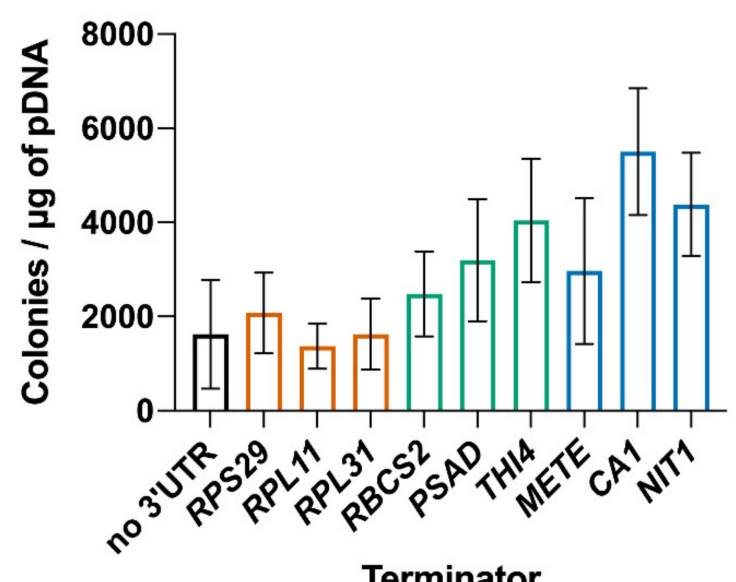

(c)

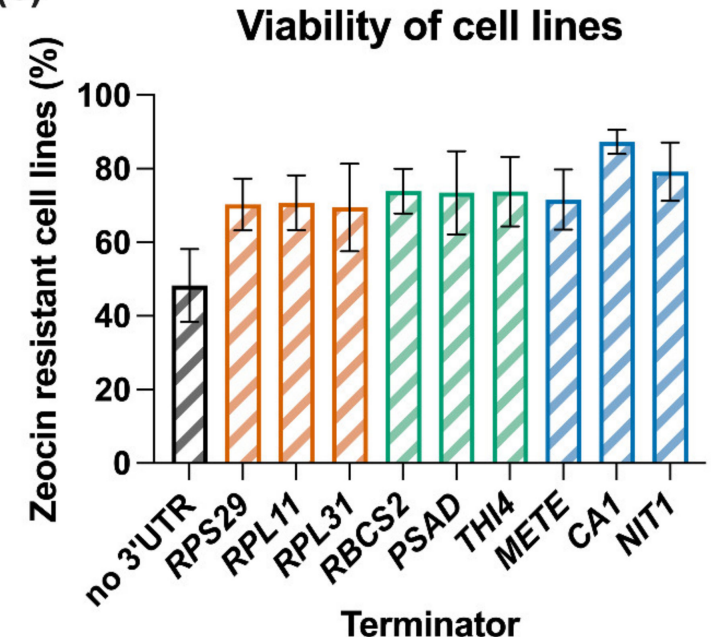

(b) Stability of expression

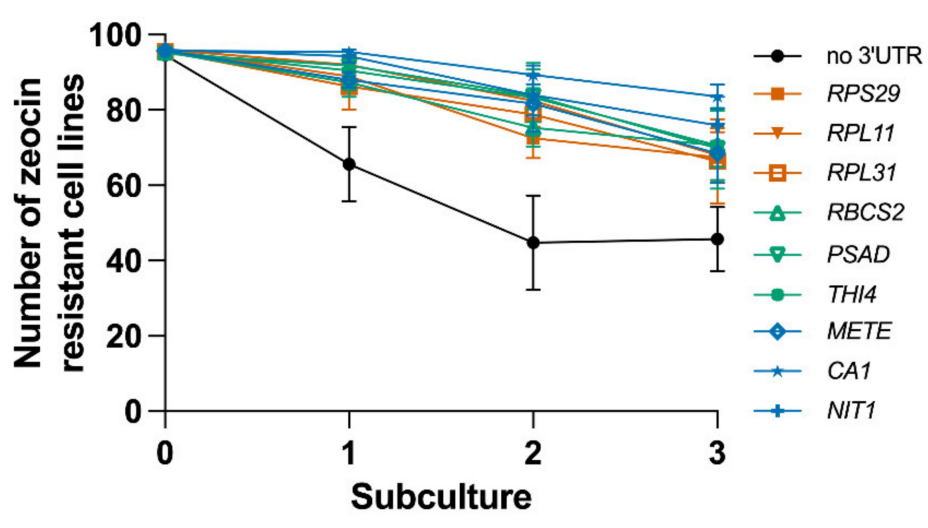

(d)

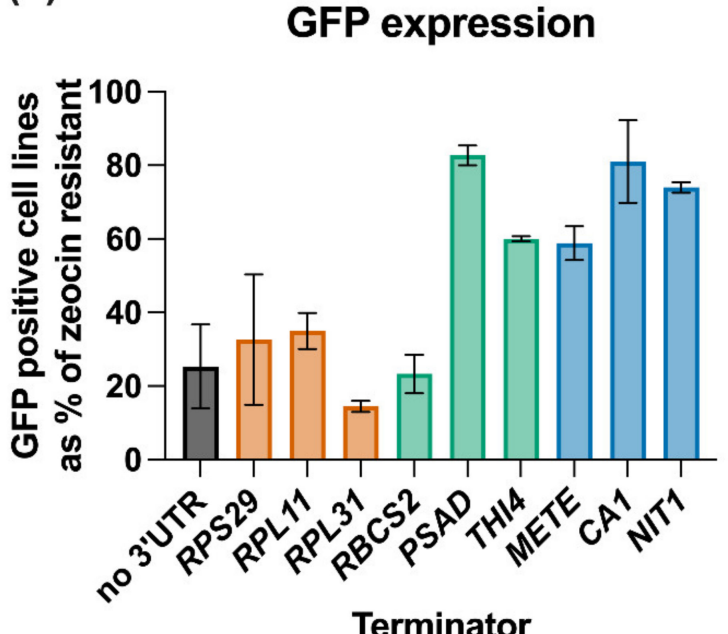

Figure 3. Analysis of the impact of terminators on transgene expression in C. reinhardtii cw15. C. reinhardtii was transformed with DNA constructs to test nine different terminators and a no-3' UTR control. (a) Transformation efficiency presented is the average of 3 replicate experiments, each containing a technical replicate $(n=6)$. (b) Stability of transformants over 3 subcultures grown for seven days under constant selective pressure derived from three independent experiments, completed in duplicates, from which 96 independent transformants were analysed per experiment and construct. (c,d) Barplot of the percentage of (c) zeocin resistant cell lines at day seven of the third subculture and (d) barplot of the percentage of zeocin-resistant (ie viable) cell lines at day that were expressing GFP above the threshold measured by confocal microscopy. A subset of $>30$ individual cell lines per $3^{\prime}$ UTR were analysed. Error bars for all charts represent standard error of mean.

\subsection{Activity of Terminators in Different C. reinhardtii Host Strains}

Host selection can have an influence on transgene expression. Cell wall deficient strains like the cw15 strain have been widely used to express transgenes, as they are more amenable to transformation compared to strains with an intact cell wall $[58,59]$. Strains UVM4 and its allelic variant UVM11, derived from a UV mutagenesis screen of a cw15 strain, exhibited enhanced expression of transgenes, accumulation of translated proteins and improved stable inheritance of the transgene over several generations [31]. However, cell wall-deficient strains have reduced motility and thus ability to mate compared to wildtype strains with an intact cell wall. In addition, upstream or downstream applications 
and limitations often make it necessary to use $C$. reinhardtii walled strains for the expression of transgenes since they are much less susceptible to shear or osmotic stress.

To determine whether the influence of terminators on transgene expression is dependent on strain selection, we tested a subset of the terminator constructs (no- $3^{\prime}$ UTR, RPS29, PSAD and CA1) in two other C. reinhardtii strains, wildtype 12 (WT12) and UVM4, as well as the previously used cw15. The same transformation protocol was used for all three strains. Colonies appeared on selection plates after 7 to 10 days for cw 15 and UVM4 transformed cell and after 15 to 20 days for WT12 transformed cells. As expected, the overall transformation efficiency in the cell-walled strain WT12 was lower than for cw15 and UVM4. Nonetheless, the relative effects of the different terminators on transformation efficiencies were similar in all three host strains (Figure 4a). Compared to the no-3' UTR control, the terminators of PSAD and CA1 produced a statistically significant $(p<0.01)$ higher number of zeocin selected transformants in each of the tested background strains. The RPS29 terminator also gave more transformants compared to the no-3' UTR control, although the difference was not statistically significant $(p>0.05)$.

A similar pattern in transgene stability, as measured by loss of zeocin resistance over time, was also seen for the different terminators across the three host strains (Figure $4 b, c)$. The decline was most pronounced in the no-3' UTR and RSP29 cell lines, whereas the CA1 and $P S A D$ lines retained a level of zeocin resistance of over $75 \%$ after three subcultures. Consistent with a previous report [31], we observed that transgenes expressed in the UVM4 strain showed a higher stability, measured as resistance to zeocin, compared to the same constructs expressed in a WT12 or cw15 strain. For three of the four cell lines (no-3' UTR, RPS29 and CA1), this higher transgene stability is statistically significant between strains $(p<0.05)$ (Figure $4 \mathrm{~b}, \mathrm{c})$.

Analysing the stable zeocin-resistant cell lines for GFP expression, again we observed the same trend across the three different strains. The percentage of cell lines expressing GFP is lower for strains transformed with constructs carrying no-3' UTR or the short RPS29 element in all three background strains, than for those using the PSAD or CA1 terminators $(p<0.01)$ (Figure $4 \mathrm{~d})$. In all three background strains over $80 \%$ of the viable cells expressed GFP when either a PSAD or CA1 terminator was used, with no statistical differences between them. In contrast, with the short RPS29 terminator, the percentage of GFP positive cell lines dropped to $52 \%$ in the UVM4 background and to $32 \%$ and $28 \%$ in cw15 and WT12, respectively. Compared to the no-3' UTR control, the short RPS29 terminator has no significant influence on the percentage of GFP positive lines $(p>0.05)$. This further reinforces the point that choice of terminator is important for stable and high transgene expression, independent of the $C$. reinhardtii host strain.

\subsection{Analysis of the Effect of Promoter Choice on Transgene Expression}

Previous work had indicated that promoter/terminator interactions can influence recombinant protein production [39], so the CA1 and PSAD terminators were combined with two additional promoters, from the PSAD and METE genes, together with their $5^{\prime}$ UTRs, and their behaviour compared with that using the original AR promoter (Figure 5). PSAD is considered a strong promoter, although it shows a bias for expression during the light period compared to the dark (Table S4; [36]), and METE is repressed by the addition of vitamin $B_{12}$ and so has the potential to be a regulatory genetic element for biotechnology purposes [56]. Plasmids were constructed, linearised and transformed into C. reinhardtii cw15 as described previously. We decided to test promoter-terminator combinations in cw15 strains only as the PSAD and CA1 terminator in combination with the $A R$ promoter were comparable between strains (Figure 4). Constructs using the PSAD promoter with either the CA1 or PSAD terminator increased transformation efficiency 1.4 and 2.8-fold $(p<0.05)$ respectively, when compared to the corresponding $A R$ promoter/terminator line (Figure 5a). In contrast, those with the METE promoter showed reduced transformation efficiency compared to $A R$, to just 17 and $39 \%$ with $C A 1$ and $P S A D$ terminators, respectively $(p<0.05)$. 


\section{(a) Transformation efficiency}

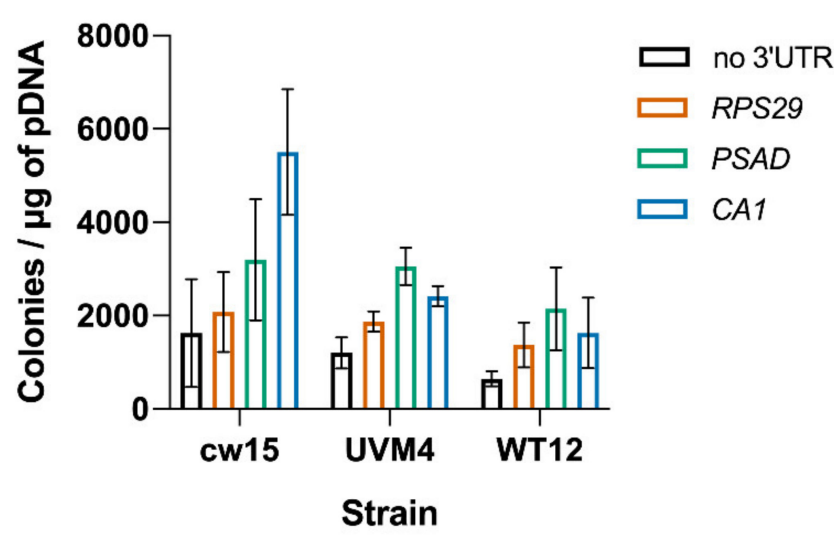

(b)

Stability of expression:
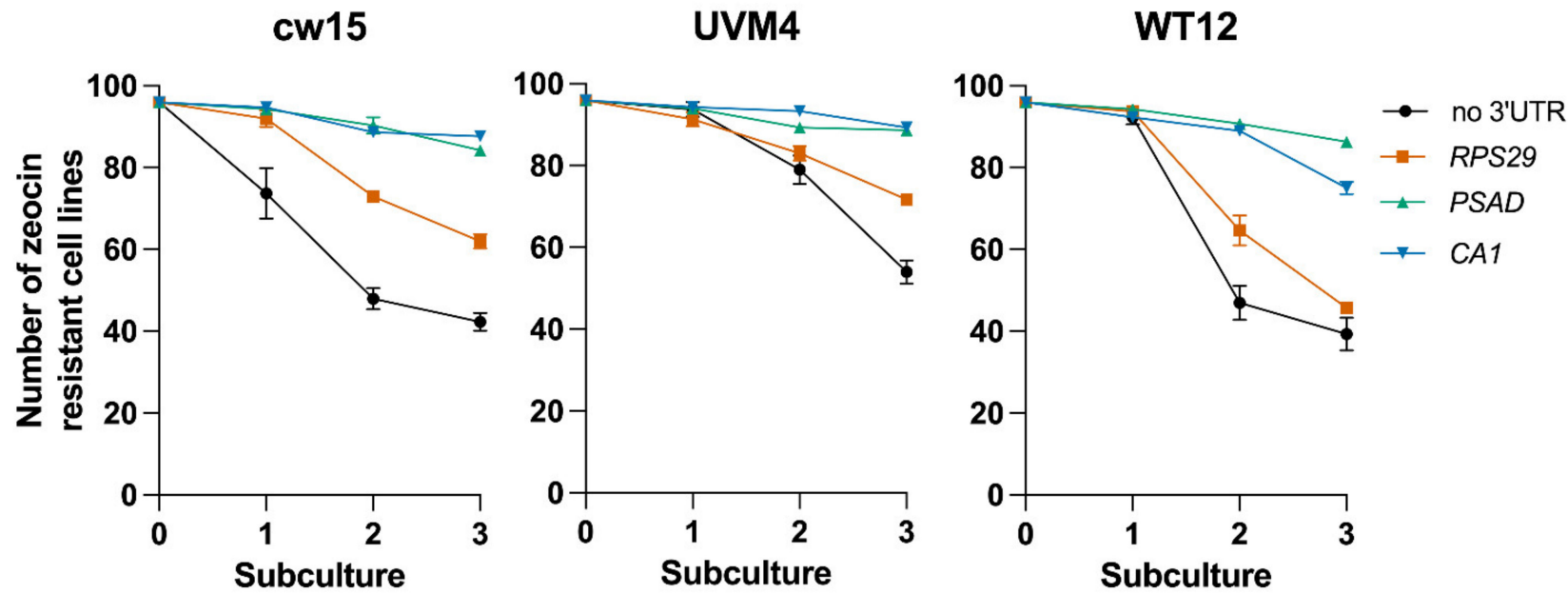

(c)

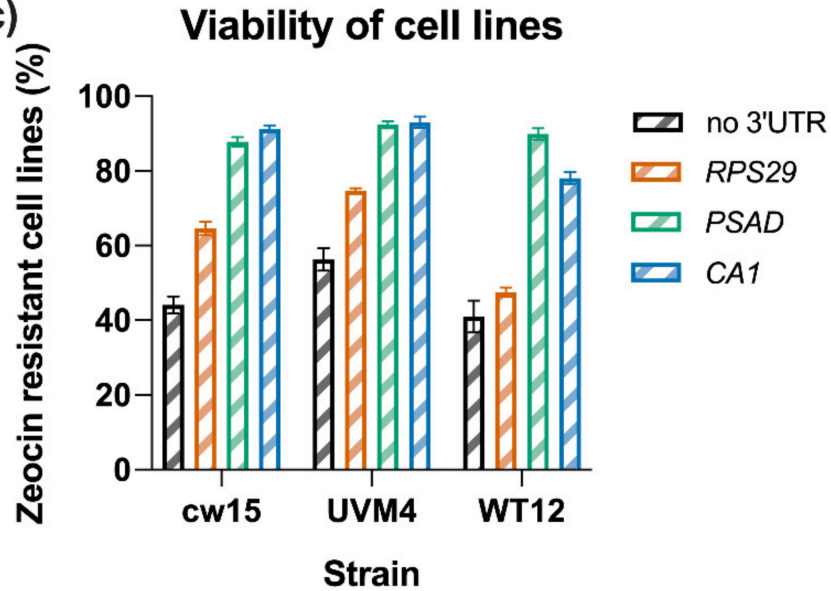

(d)

\section{GFP expression}

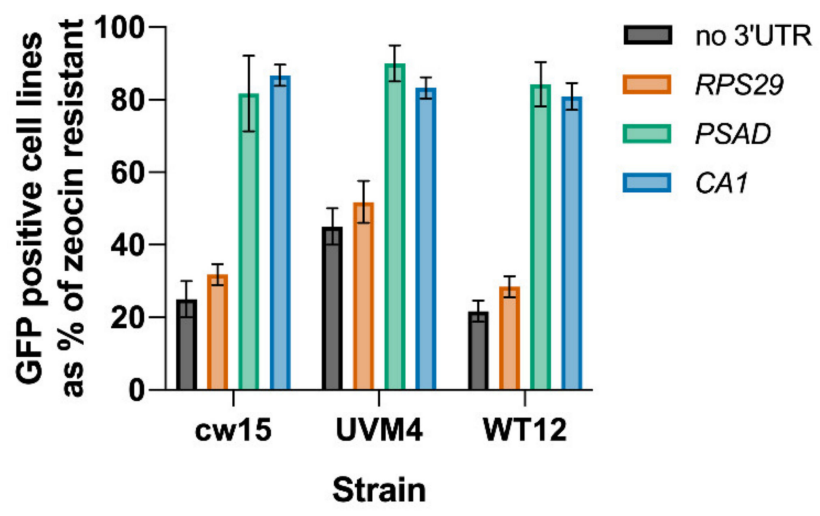

Figure 4. Influence of terminators on transgene expression in different $C$. reinhardtii strains. (a) Transformation efficiency for constructs carrying three different terminators (RPS29, PSAD, CA1) transformed into different $C$. reinhardtii strains: the cell wall deficient strains cw15 and UVM4, and the walled strain WT12. Presented are the averages of three replicate experiments. (b) Stability of ble-gene expression over three subcultures under constant antibiotic selection is shown for cw15 (left), UVM4 (middle) and WT12 (right panel). (c,d) Percentage of zeocin-resistant cell lines at day seven of the third subculture and (d) percentage of those zeocin resistant cell lines expressing GFP above the threshold detectable by confocal microscopy. Error bars for all charts represent standard error of mean. Data for strain cw15 is also shown in Figure 3. 
(a) Transformation efficiency

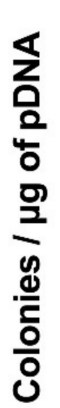

1500

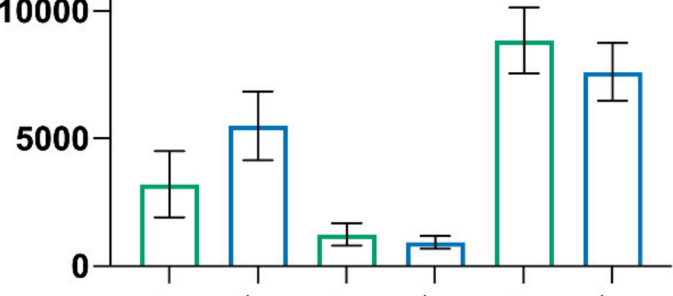

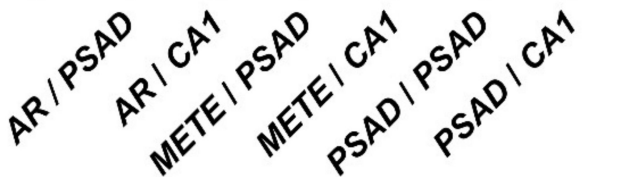

Promoter / Terminator combination

(c)

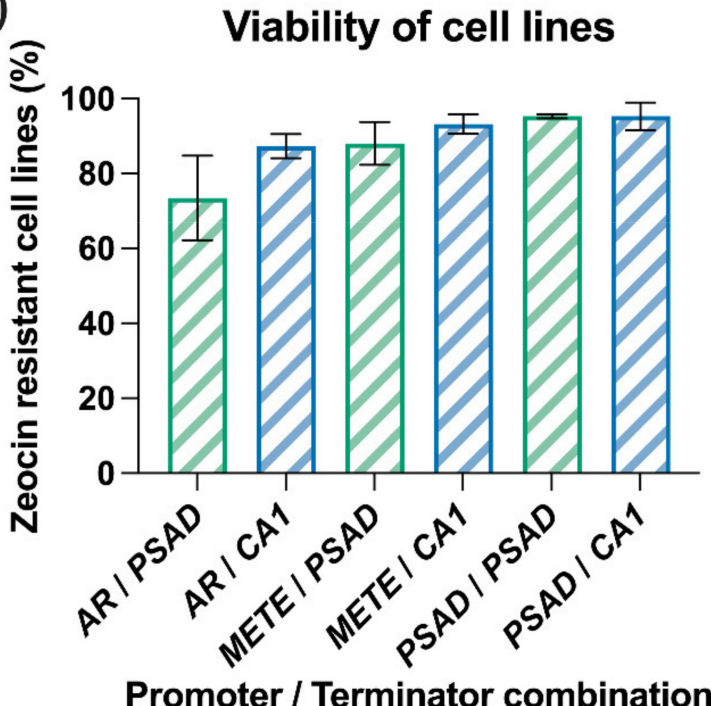

(b) Stability of expression

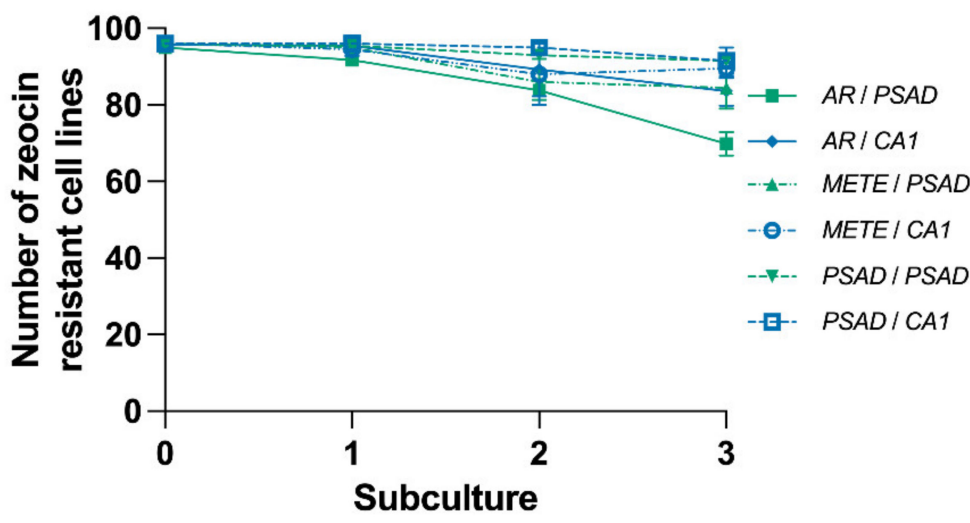

(d) GFP expression

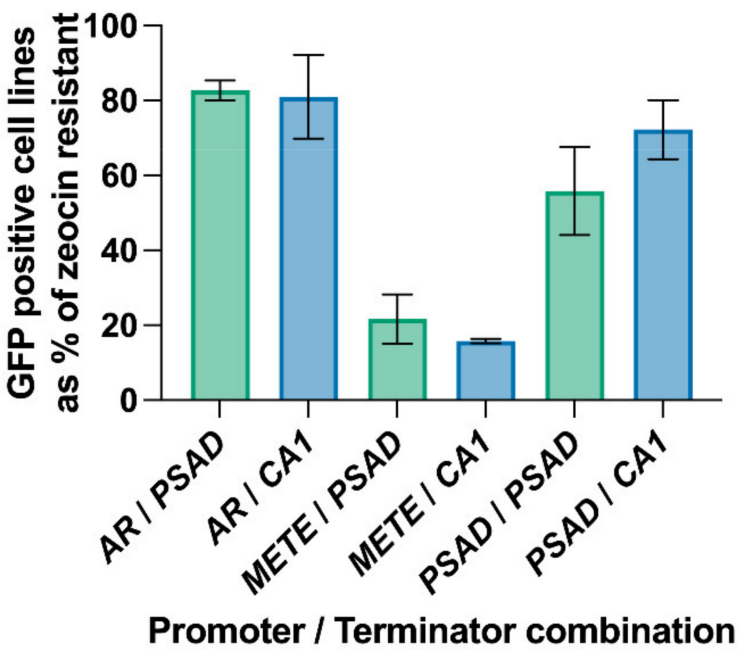

Figure 5. Analysis of the impact of different promoter and terminator combinations on transgene expression. The cell wall deficient strain cw15 was transformed with DNA construct expressing the Ble-GFP reporter under the control of different promoter $/ 5^{\prime}$ UTR and terminator combinations. The three promoters $A R, M E T E$ and PSAD were combined with the CA1 or PSAD terminator. (a) Transformation efficiency for transformants carrying the different promoter/terminator combinations. Presented are the averages of three replicate experiments. (b) Expression stability of transformants over three subcultures under constant antibiotic selection. (c,d) Percentage of zeocin resistant cell lines at day seven of the third subculture that were also GFP-positive as determined by confocal microscopy. Error bars for all charts represent standard error of mean.

Nonetheless, both the PSAD and METE promoter cell lines, when employed with the terminator of $P S A D$, exhibited respectively $95 \%$ and $88 \%$ stability after three subcultures (Figure 5b,c). Comparable constructs with the CA1 terminator showed a similar stability with both the PSAD and METE promoters. In the lines transformed with the $A R$ promoter / no- $3^{\prime}$ UTR construct only $\sim 45 \%$ of initial picked cell lines remained zeocin resistant (Figure 3). Compared to this control, the percentage of zeocin viable cell lines is significant higher in all other tested promoter/terminator combinations $(p<0.05)$.

To investigate the influence of the promoter on threshold gene expression, confocal microscopy was used to test for GFP expression on a subset of the stable transformed 
cell lines. When the promoter was changed from $A R$ to PSAD no significant change in the frequency of GFP positives in the sample set was observed when the CA1 terminator was used. However, there was a significant reduction with the PSAD promoter/PSAD terminator combination, from $83 \%$ to $55 \%(p<0.05)$ (Figure $5 \mathrm{~d}$ ). Using the METE promoter produced an even greater reduction in the frequency of GFP positives in the sample, down to $16 \%$ with the CA1 element $(p<0.0005)$ and $22 \%$, with the PSAD terminator $(p<0.0005)$ (Figure 5d). These results might reflect the fact that the METE promoter element used here is noticeably weaker when controlling transgenes than the level of native METE transcript, possibly because other elements of the gene are necessary for high level expression [56,60].

\subsection{Testing Promoter and Terminator Combinations to Drive Expression of a Selection} Marker-Independent Transgene

So far, the analysis of the terminators has been with a single transgene expressing the fusion protein Ble-GFP, which acted as both the selection marker and the reporter protein. We wanted to examine the influence of terminators on this as a reporter protein per se, by using a different marker to select the initial transformants. Ble-GFP under the control of the PSAD promoter and the three terminators used earlier, RPS29, PSAD and $C A 1$ was cloned together with the paromomycin resistance gene AphVIII as the selection marker, driven by the $A R$ promoter/PSAD terminator. Using the same reporter, Ble-GFP ensured that the results could be compared directly with previous experiments (Figure 6a). This experimental design mimics metabolic engineering applications, where cassettes for introduced biosynthetic enzymes would be used together with a separate selection marker $[52,61,62]$. To allow the combinatorial assembly of different promoter/terminator combinations, Golden Gate assembly using parts from the Chlamydomonas MoClo kit was used [18]. We included the intron $R B C S 2 i 1$ upstream of the gene coding regions to enhance transgene expression [13]. It should also be noted that the PSAD terminator L0 part in the MoClo kit is $548 \mathrm{bp}$ long, similar to the one used in previous studies [39], rather than the $366 \mathrm{bp}$ sequence used in the initial experiments (Figures 3-5).

UVM4 was transformed with the different constructs and transformants were selected on plates containing paromomycin, but not zeocin. As expected, no difference in transformation efficiencies was observed with any construct (Figure 6 b) because the selection was based on the same marker. Similarly, subculturing the primary transformants in TAP media with paromomycin and scoring growth to determine levels of AphVIII transgene expression, showed excellent stability over time as would be expected for the PSAD terminator, with over $85 \%$ of the initial cells still paromomycin resistant (Figure $6 c, d$ ). After the third subculture, the paromomycin resistant cell lines were analysed for expression of the Ble-GFP transgene from the independent expression cassette, scoring both zeocin resistance and GFP expression. When the short terminator RPS 29 was used 59\% of the tested cell lines were zeocin resistant and $43 \%$ expressed GFP above detectable limits of the confocal microscope (Figure $6 \mathrm{e}, \mathrm{f})$. The percentage was significantly increased when using the PSAD terminator ( $81 \%$ and $80 \%$ ) or the CA1 terminator ( $78 \%$ and $72 \%$ ) for zeocin resistance or GFP fluorescence respectively $(p<0.005)$. Again, this demonstrates that the longer terminators PSAD and CA1 are more effective than the short RPS29. When transforming the nuclear genome with a construct containing independent expression and selection cassettes, colonies with the integrated selection marker, but not expression cassette, may arise because of the DNA fragmentation by endonuclease cleavage during electroporation [28]. As up to $80 \%$ of paromomycin resistant lines were also zeocin resistant, these lines contained both the expression and the selection cassettes integrated into the genome, so the fragmentation of the transformation cassette was $<20 \%$. 
(a) Expression cassette Selection cassette

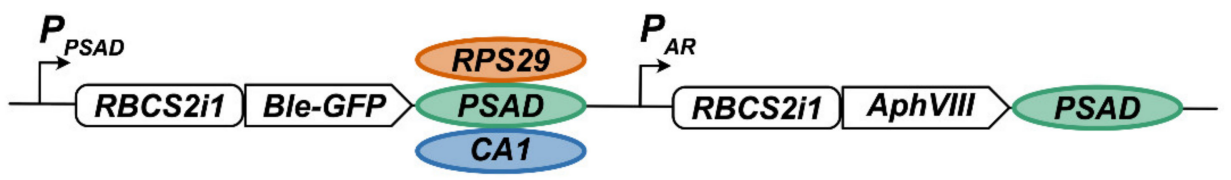

(b) Transformation efficiency (c) Stability of expression
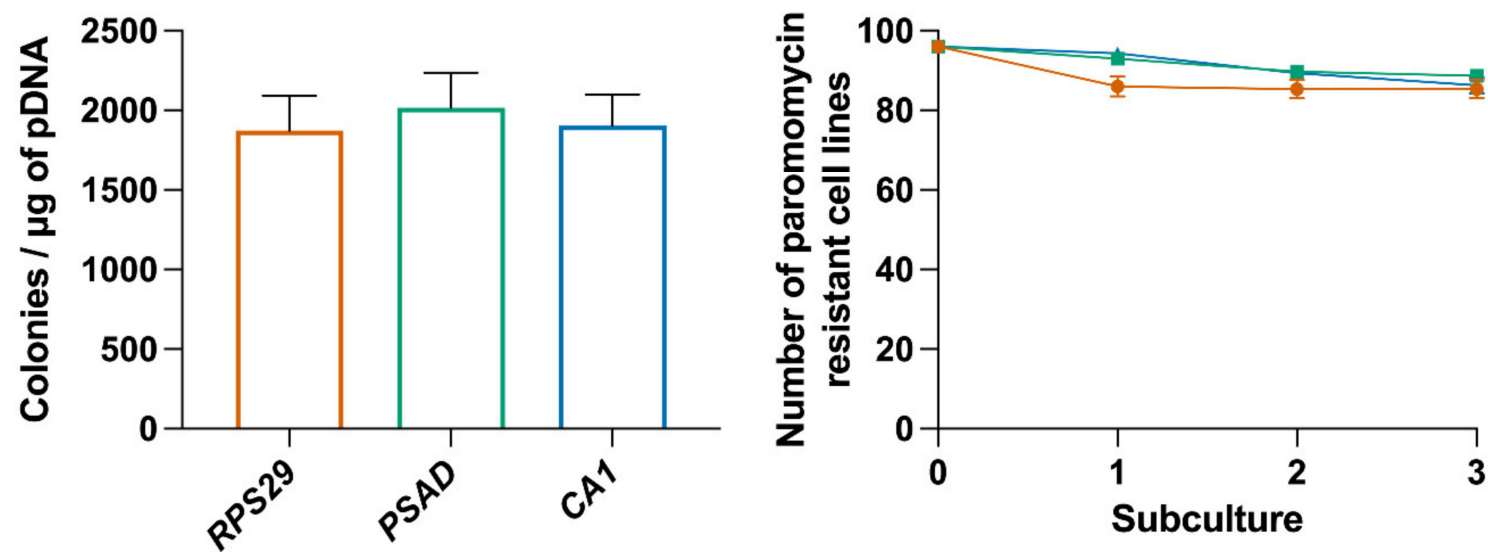

(d)
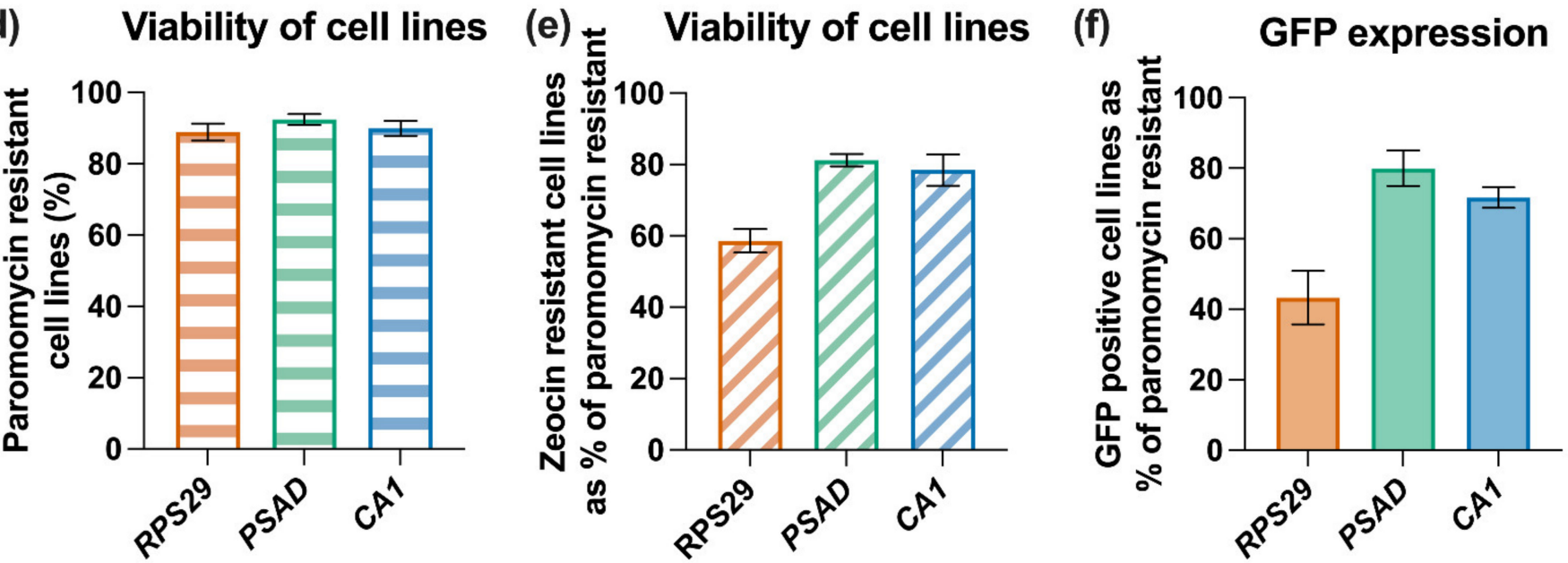

Figure 6. Testing terminators driving expression of transgenes in two different expression cassettes. (a) Schematic of construct design using two expression cassettes. The selection cassette is driving expression of the paromomycin resistance gene AphVIII using an AR promoter / PSAD terminator combination. In the Ble-GFP expression cassette the PSAD promoter is driving expression and three different terminators are tested: RPS29, PSAD and CA1. Constructs were introduced into strain UVM4. (b) transformation efficiency for the average of 3 replicate experiments, each containing a technical replicate $(n=6)$. (c) Stability of transformants over 3 subcultures under constant antibiotic selection of paromomycin. (d) Barplot of the percentage of paromomycin resistant cell lines derived from the experiment. (e) Barplot of zeocin re-sistant cell lines from the experiment. (f) Barplot of GFP positive cell lines from the experiment.

\section{Discussion}

In the work presented here we used a standardised workflow to demonstrate quantitatively the influence of nine different terminators on transgene expression in C. reinhardtii. We found that the terminators from $P S A D$ and $C A 1$ gave the best results in all experiments. The effectiveness of the PSAD terminator for transgene expression in $C$. reinhardtii has been noted previously $[18,39,40]$ although the reason for this is unknown. In contrast, the short terminators $(<100 \mathrm{bp})$ of the three ribosomal subunits we tested, RPL11, RPL31 and 
RPS29, performed much less well in terms of transformation efficiency, transgene stability and level of expression than did the longer terminators (Figure 3), and were actually no better in terms of expression of GFP above the threshold than the negative control (no- $3^{\prime}$ UTR), which had no defined sequence after the stop codon. This is despite the fact that the ribosomal subunits are more highly expressed across the diurnal cycle (Table 1; [36]) than the other genes. Similarly, we found that the $221 \mathrm{bp}$ terminator element from the RBCS2 gene, whose expression levels are ranked even higher than the ribosomal genes, was less effective, particularly in terms of threshold expression of the GFP reporter, than the other five elements (with lengths from 336 to $888 \mathrm{bp}$ ), even though all transgenes were driven by the same chimeric HSP70A/ $\underline{R} B C S 2$ promoter (AR). Conversely, the NIT1 terminator was effective in driving reporter gene levels above the threshold in the majority of stable transformants, despite its low expression ranking under the conditions of cell growth (in media containing ammonium ions and no nitrate). This lack of correlation between expression level of the native gene and the ability of its regulatory elements to confer similar properties on a transgene is consistent with the earlier observation by López-Paz et al. [40] on two other ribosomal proteins: the RPL23 terminator was effective in promoting high level expression, but that for RPL35a was not, whilst their expression levels were both in the top rank in the diurnal RNA-seq dataset [63].

The behaviours observed in the first experiment (Figure 3) were recapitulated when a subset of the terminators was tested in different host strains (Figure 4). Transformants with the PSAD and CA1 terminators showed stable and high-level expression irrespective of the host, whereas RPS29 performed the same or only slightly better than the no-3' UTR negative control. Stability of the zeocin-resistance phenotype in transformants was better in strain UVM4 than cw15 or WT12 as expected [31], particularly for those carrying the $P S A D$ and CA1 terminators (Figure $4 \mathrm{~b}$ ), but there was no discernible difference in the proportion of transformants expressing GFP above a threshold (Figure 4d). Exchanging the $A R$ promoter for that from PSAD again demonstrated that the PSAD and CA1 terminators conferred much better stability and expression of the transgene than the no- $3^{\prime}$ UTR negative control (Figure 5). When the METE promoter was used only an increase in stability was observed, with little or no GFP expression above baseline. This is likely to reflect the fact that METE promoter element used here is much weaker than PSAD or AR [56], so that whilst there is sufficient Ble protein to confer zeocin-resistance, GFP fluorescence is at or below the minimum detection threshold. In this context, it should be emphasised that these data are not the absolute level of GFP expression, simply an indication of whether or not fluorescence was observed by confocal microscopy. When the RPS29, PSAD and CA1 terminators were used to regulate the transgene separately from the selectable marker they again behaved as predicted (Figure 6). Together, these results indicate that the terminator parts are acting in an orthogonal manner, meaning that they could be expected to behave similarly in other construct designs. Indeed, we have successfully used the CA1 terminator to express casbene synthase from the higher plant Jatropha curcas in C. reinhardtii [52]; the engineered strain is still producing the diterpene casbene at the same level over 2 years later.

Our aim in this work has been to enhance the already extensive synthetic biology toolkit for C. reinhardtii to increase the flexibility for transgene expression in this organism. The advantage of the approach we have taken here to screen for optimal genetic parts, in this case terminator elements, is that with a defined workflow, we were able to explore the parameter space across a range of different characteristics systematically and quantitatively. The method is relatively rapid ( $4-5$ weeks from transformation to output on the activity of the parts). To facilitate visualisation of the results that we collected and allow an objective assessment of which would be the most suitable to use in subsequent construct designs, we combined them in Figure 7, plotting the stability of the zeocin-resistance against the proportion of the stable lines expressing GFP above the threshold. The size of the circles represents the transformation efficiency. In Figure 7a, all the data from cw15 are presented, whereas in Figure $7 b$, the data available from all three strains are shown. There is a clear trend of a positive correlation between the three parameters that are quantified, with the 
constructs containing the PSAD and CA1 terminators clustering together in the top right of the graphs. Thus, even though differences in the measured parameters are relatively small, combining the measurements provides more confidence in the choice of optimal parts for any particular application.

(a)

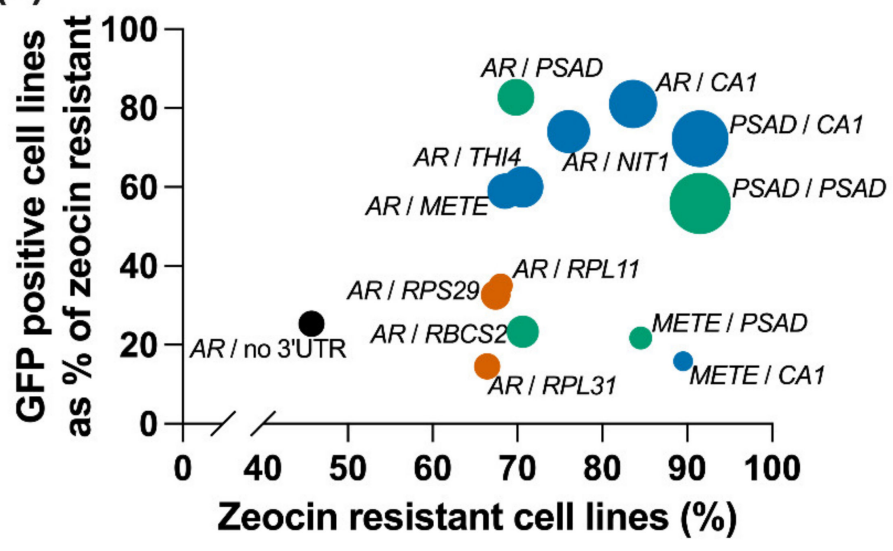

(b)

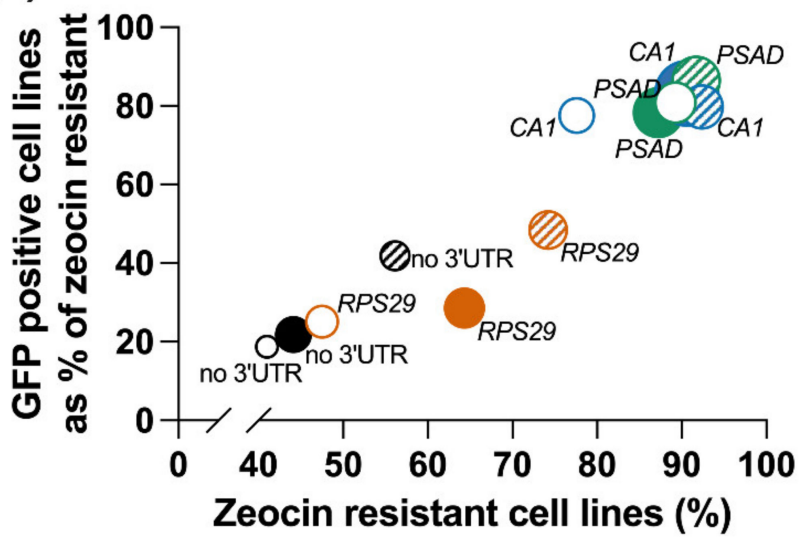

Figure 7. Impact of terminators on different parameters to measure transgene expression in C. reinhardtii. (a) The influence of different promoter/terminator combinations on transformation efficiency (circle size), resistance to zeocin ( $x$-axis) and GFP expression ( $y$-axis) tested in C. reinhardtii cw15. (b) The effect of a subset of terminators (RPS29, PSAD, CA1) on transformation efficiency (circle size), resistance to zeocin ( $x$-axis) and GFP expression over the detection limit ( $y$-axis) when tested in different C. reinhardtii strains, cw15 transformants (filled circles), UVM4 transformants (hatched circles) and WT12 transformants (open circles). Shown are average data from three independent transformation experiments.

In summary, we have expanded the set of terminators that can be used for stable and high transgene expression in C. reinhardtii. The $C A 1$ terminator behaves very similarly to the well-characterised $P S A D$, and so offers the means to use distinct but effective terminators for the selectable marker and the transgene in a typical construct design. Both of these are part of the original MoClo kit [18], which enables rapid assembly via modular cloning. In addition, three other terminators, from the NIT1, METE and THI4 genes, have been shown to be effective, and they too have been domesticated for use in the same system and syntax. All are available from the Chlamydomonas Resource Centre [19].

Supplementary Materials: The following are available online at https:/ / www.mdpi.com/article/ 10.3390/life11090964/s1, Figure S1: Comparison of no selection versus selection workflow, Figure S2: Analysis of genomic features in C. reinhardtii., Table S1: Primers used for Gibson assembly and DNA part generation, Table S2: List of plasmids generated by Gibson assembly, Table S3: MoClo constructs employed and generated, Table S4: Expression ranking of C. reinhardtii genes used in this study, Dataset S1: Distribution of genomic elements in C. reinhardtii genome, Dataset S2: Gene expression values from Strenkert et al.

Author Contributions: Conceptualization, A.G.S.; methodology, K.G., M.A.S. and A.H.; formal analysis, K.G., M.A.S. and A.H.; investigation, K.G., M.A.S., A.H., E.V.T. and P.M.; data curation, K.G., M.A.S. and A.H.; writing—original draft preparation, K.G.; writing-review and editing, K.G., P.M., P.M.M., G.I.M.O., A.H. and A.G.S.; visualization, A.H. and P.M.M.; supervision, A.G.S.; project administration, A.G.S.; funding acquisition, A.G.S. All authors have read and agreed to the published version of the manuscript.

Funding: This research was funded by BBSRC grants BB/I007660/1 (M.A.S. \& A.G.S.), BB/L002957/1 (K.G. \& A.G.S.), BB/R01860X/1 (P.M.M. \& A.G.S.), BB/L014130/1 (G.I.M.O., P.M., K.G. \& A.G.S.) and BB/M018180/1 (P.M. \& A.G.S.), Bill \& Melinda Gates Foundation grant OPP1144 (A.H.) and EU FP7 Collaborative Project SPLASH Grant number 311956 (M.A.S. \& A.G.S.).

Institutional Review Board Statement: Not applicable. 
Informed Consent Statement: Not applicable.

Data Availability Statement: The data is available in the data repository of the University of Cambridge, UK.

Acknowledgments: We are grateful for laboratory support from Eleanor Tomsett, who helped with data collection, Faye Loo, who helped with cloning of DNA constructs, Fiona Taylor and Katie Sutherland for algal subculturing and Sue Aspinall and Lorraine Archer for laboratory management. C. reinhardtii strain UVM4 was obtained from Ralph Bock (MPI-MP, Golm, Germany).

Conflicts of Interest: The authors declare no conflict of interest. The funders had no role in the design of the study, in the collection, analyses, or interpretation of data, in the writing of the manuscript, or in the decision to publish the results.

\section{References}

1. Sproles, A.E.; Fields, F.J.; Smalley, T.N.; Le, C.H.; Badary, A.; Mayfield, S.P. Recent advancements in the genetic engineering of microalgae. Algal Res. 2021, 53, 102158. [CrossRef]

2. Eberhard, S.; Finazzi, G.; Wollman, F.-A. The dynamics of photosynthesis. Annu. Rev. Genet. 2008, 42, 463-515. [CrossRef]

3. Wingfield, J.L.; Lechtreck, K.-F. Chlamydomonas basal bodies as flagella organizing centers. Cells 2018, 7, 79. [CrossRef] [PubMed]

4. Scaife, M.A.; Nguyen, G.T.; Rico, J.; Lambert, D.; Helliwell, K.; Smith, A. Establishing Chlamydomonas reinhardtii as an industrial biotechnology host. Plant J. 2015, 82, 532-546. [CrossRef]

5. Jinkerson, R.; Jonikas, M.C. Molecular techniques to interrogate and edit the Chlamydomonas nuclear genome. Plant J. 2015, 82, 393-412. [CrossRef]

6. Salomé, P.A.; Merchant, S.S. A series of fortunate events: Introducing Chlamydomonas as a reference organism. Plant Cell 2019, 31, 1682-1707. [CrossRef]

7. Harris, E.H.; Stern, D.B.; Witman, G.B. (Eds.) The Chlamydomonas Sourcebook, 2nd ed.; Academic Press: Cambridge, MA, USA, 2009; ISBN 978-0-12-370873-1.

8. Merchant, S.S.; Prochnik, S.E.; Vallon, O.; Harris, E.H.; Karpowicz, S.J.; Witman, G.B.; Terry, A.; Salamov, A.; Fritz-Laylin, L.K.; Maréchal-Drouard, L.; et al. The Chlamydomonas genome reveals the evolution of key animal and plant functions. Science 2007, 318, 245-250. [CrossRef]

9. Tran, N.T.; Kaldenhoff, R. Achievements and challenges of genetic engineering of the model green alga Chlamydomonas reinhardtii. Algal Res. 2020, 50, 101986. [CrossRef]

10. Shimogawara, K.; Fujiwara, S.; Grossman, A.; Usuda, H. High-efficiency transformation of Chlamydomonas reinhardtii by electroporation. Genetics 1998, 148, 1821-1828. [CrossRef] [PubMed]

11. Kindle, K.L. High-frequency nuclear transformation of Chlamydomonas reinhardtii. Proc. Natl. Acad. Sci. USA 1990, 87, 1228-1232. [CrossRef]

12. Fuhrmann, M.; Oertel, W.; Hegemann, P. A synthetic gene coding for the green fluorescent protein (GFP) is a versatile reporter in Chlamydomonas reinhardtii. Plant J. 1999, 19, 353-361. [CrossRef]

13. Sizova, I.; Fuhrmann, M.; Hegemann, P. A Streptomyces rimosus aphVIII gene coding for a new type phosphotransferase provides stable antibiotic resistance to Chlamydomonas reinhardtii. Gene 2001, 277, 221-229. [CrossRef]

14. Rasala, B.A.; Barrera, D.J.; Ng, J.; Plucinak, T.M.; Rosenberg, J.N.; Weeks, N.P.; Oyler, G.A.; Peterson, T.C.; Haerizadeh, F.; Mayfield, S.P. Expanding the spectral palette of fluorescent proteins for the green microalga Chlamydomonas reinhardtii. Plant J. 2013, 74, 545-556. [CrossRef] [PubMed]

15. Rohr, J.; Sarkar, N.; Balenger, S.; Jeong, B.-R.; Cerutti, H. Tandem inverted repeat system for selection of effective transgenic RNAi strains in Chlamydomonas. Plant J. 2004, 40, 611-621. [CrossRef] [PubMed]

16. Molnar, A.; Bassett, A.; Thuenemann, E.; Schwach, F.; Karkare, S.; Ossowski, S.; Weigel, D.; Baulcombe, D. Highly specific gene silencing by artificial microRNAs in the unicellular alga Chlamydomonas reinhardtii. Plant J. 2009, 58, 165-174. [CrossRef]

17. Ferenczi, A.; Pyott, D.E.; Xipnitou, A.; Molnar, A. Efficient targeted DNA editing and replacement in Chlamydomonas reinhardtii using Cpf1 ribonucleoproteins and single-stranded DNA. Proc. Natl. Acad. Sci. USA 2017, 114, 13567-13572. [CrossRef] [PubMed]

18. Crozet, P.; Navarro, F.J.; Willmund, F.; Mehrshahi, P.; Bakowski, K.; Lauersen, K.; Pérez-Pérez, M.-E.; Auroy, P.; Rovira, A.G.; Sauret-Gueto, S.; et al. Birth of a photosynthetic chassis: A MoClo toolkit enabling synthetic biology in the microalga Chlamydomonas reinhardtii. ACS Synth. Biol. 2018, 7, 2074-2086. [CrossRef]

19. Chlamydomonas Resource Center. Available online: https://www.chlamycollection.org (accessed on 10 September 2021).

20. Rasala, B.A.; Mayfield, S.P. Photosynthetic biomanufacturing in green algae; production of recombinant proteins for industrial, nutritional, and medical uses. Photosynth. Res. 2014, 123, 227-239. [CrossRef]

21. Dyo, Y.M.; Purton, S. The algal chloroplast as a synthetic biology platform for production of therapeutic proteins. Microbiology 2018, 164, 113-121. [CrossRef]

22. Taunt, H.N.; Stoffels, L.; Purton, S. Green biologics: The algal chloroplast as a platform for making biopharmaceuticals. Bioengineered 2018, 9, 48-54. [CrossRef] 
23. Rasala, B.A.; Chao, S.-S.; Pier, M.; Barrera, D.J.; Mayfield, S.P. Enhanced genetic tools for engineering multigene traits into green algae. PLoS ONE 2014, 9, e94028. [CrossRef]

24. Chlamydomonas Spatial Interactome. Available online: https:// sites.google.com/site/chlamyspatialinteractome (accessed on 10 September 2021).

25. Mackinder, L.C.; Chen, C.; Leib, R.D.; Patena, W.; Blum, S.R.; Rodman, M.; Ramundo, S.; Adams, C.M.; Jonikas, M.C. A spatial interactome reveals the protein organization of the algal $\mathrm{CO}_{2}$-concentrating mechanism. Cell 2017, 171, 133-147. [CrossRef] [PubMed]

26. Rosales-Mendoza, S.; Paz-Maldonado, L.M.T.; Soria-Guerra, R.E. Chlamydomonas reinhardtii as a viable platform for the production of recombinant proteins: Current status and perspectives. Plant Cell Rep. 2011, 31, 479-494. [CrossRef]

27. Lauersen, K.J.; Wichmann, J.; Baier, T.; Kampranis, S.; Pateraki, I.; Møller, B.L.; Kruse, O. Phototrophic production of heterologous diterpenoids and a hydroxy-functionalized derivative from Chlamydomonas reinhardtii. Metab. Eng. 2018, 49, 116-127. [CrossRef]

28. Zhang, R.; Patena, W.; Armbruster, U.; Gang, S.; Blum, S.R.; Jonikas, M.C. High-throughput genotyping of green algal mutants reveals random distribution of mutagenic insertion sites and endonucleolytic cleavage of transforming DNA. Plant Cell 2014, 26, 1398-1409. [CrossRef]

29. Cerutti, H.; Johnson, A.M.; Gillham, N.W.; Boynton, J.E. Epigenetic silencing of a foreign gene in nuclear transformants of Chlamydomonas. Plant Cell 1997, 9, 925-945. [CrossRef]

30. Schroda, M. Good news for nuclear transgene expression in Chlamydomonas. Cells 2019, 8, 1534. [CrossRef] [PubMed]

31. Neupert, J.; Karcher, D.; Bock, R. Generation of Chlamydomonas strains that efficiently express nuclear transgenes. Plant J. 2009, 57, 1140-1150. [CrossRef]

32. Neupert, J.; Gallaher, S.D.; Lu, Y.; Strenkert, D.; Segal, N.; Barahimipour, R.; Fitz-Gibbon, S.T.; Schroda, M.; Merchant, S.S.; Bock, R An epigenetic gene silencing pathway selectively acting on transgenic DNA in the green alga Chlamydomonas. Nat. Commun. 2020, 11, 1-17. [CrossRef]

33. Barahimipour, R.; Strenkert, D.; Neupert, J.; Schroda, M.; Merchant, S.S.; Bock, R. Dissecting the contributions of GC content and codon usage to gene expression in the model alga Chlamydomonas reinhardtii. Plant J. 2015, 84, 704-717. [CrossRef]

34. Eichler-Stahlberg, A.; Weisheit, W.; Ruecker, O.; Heitzer, M. Strategies to facilitate transgene expression in Chlamydomonas reinhardtii. Planta 2009, 229, 873-883. [CrossRef] [PubMed]

35. Baier, T.; Wichmann, J.; Kruse, O.; Lauersen, K. Intron-containing algal transgenes mediate efficient recombinant gene expression in the green microalga Chlamydomonas reinhardtii. Nucleic Acids Res. 2018, 46, 6909-6919. [CrossRef] [PubMed]

36. Strenkert, D.; Schmollinger, S.; Gallaher, S.D.; Salomé, P.A.; Purvine, S.O.; Nicora, C.D.; Mettler-Altmann, T.; Soubeyrand, E.; Weber, A.; Lipton, M.S.; et al. Multiomics resolution of molecular events during a day in the life of Chlamydomonas. Proc. Natl. Acad. Sci. USA 2019, 116, 2374-2383. [CrossRef]

37. Schroda, M.; Blocker, D.; Beck, C.F. The HSP70A promoter as a tool for the improved expression of transgenes in Chlamydomonas. Plant J. 2000, 21, 121-131. [CrossRef]

38. Scranton, M.A.; Ostrand, J.T.; Georgianna, D.R.; Lofgren, S.M.; Li, D.; Ellis, R.C.; Carruthers, D.N.; Dräger, A.; Masica, D.L.; Mayfield, S.P. Synthetic promoters capable of driving robust nuclear gene expression in the green alga Chlamydomonas reinhardtii. Algal Res. 2016, 15, 135-142. [CrossRef]

39. Kumar, A.; Falcao, V.R.; Sayre, R.T. Evaluating nuclear transgene expression systems in Chlamydomonas reinhardtii. Algal Res. 2013, 2, 321-332. [CrossRef]

40. López-Paz, C.; Liu, D.; Geng, S.; Umen, J.G. Identification of Chlamydomonas reinhardtii endogenous genic flanking sequences for improved transgene expression. Plant J. 2017, 92, 1232-1244. [CrossRef]

41. De Felippes, F.F.; McHale, M.; Doran, R.L.; Roden, S.; Eamens, A.L.; Finnegan, E.J.; Waterhouse, P.M. The key role of terminators on the expression and post-transcriptional gene silencing of transgenes. Plant J. 2020, 104, 96-112. [CrossRef] [PubMed]

42. Kuehner, J.; Pearson, E.L.; Moore, C. Unravelling the means to an end: RNA polymerase II transcription termination. Nat. Rev. Mol. Cell Biol. 2011, 12, 283-294. [CrossRef]

43. Mapendano, C.K.; Lykke-Andersen, S.; Kjems, J.; Bertrand, E.; Jensen, T.H. Crosstalk between mRNA $3^{\prime}$ end processing and transcription initiation. Mol. Cell 2010, 40, 410-422. [CrossRef]

44. Andersen, P.K.; Jensen, T.H.; Lykke-Andersen, S. Making ends meet: Coordination between RNA $3^{\prime}$-end processing and transcription initiation. Wiley Interdiscip. Rev. RNA 2013, 4, 233-246. [CrossRef]

45. Wang, P.-H.; Kumar, S.; Zeng, J.; McEwan, R.; Wright, T.R.; Gupta, M. Transcription terminator-mediated enhancement in transgene expression in maize: Preponderance of the AUGAAU motif overlapping with poly (A) signals. Front. Plant Sci. 2020, 11, 570778. [CrossRef]

46. Bell, S.A.; Shen, C.; Brown, A.; Hunt, A.G. Experimental genome-wide determination of RNA polyadenylation in Chlamydomonas reinhardtii. PLoS ONE 2016, 11, e0146107. [CrossRef]

47. Davey, M.P.; Horst, I.; Duong, G.-H.; Tomsett, E.V.; Litvinenko, A.C.P.; Howe, C.J.; Smith, A. Triacylglyceride production and autophagous responses in Chlamydomonas reinhardtii depend on resource allocation and carbon source. Eukaryot. Cell 2014, 13, 392-400. [CrossRef] [PubMed] 
48. Kropat, J.; Hong-Hermesdorf, A.; Casero, D.; Ent, P.; Castruita, M.; Pellegrini, M.; Merchant, S.S.; Malasarn, D. A revised mineral nutrient supplement increases biomass and growth rate in Chlamydomonas reinhardtii. Plant J. 2011, 66, 770-780. [CrossRef] [PubMed]

49. Gibson, D.G.; Young, L.; Chuang, R.Y.; Venter, J.C.; Hutchison, C.A.; Smith, H.O. Enzymatic assembly of DNA molecules up to several hundred kilobases. Nat. Methods 2009, 6, 343-345. [CrossRef]

50. Weber, E.; Engler, C.; Gruetzner, R.; Werner, S.; Marillonnet, S. A modular cloning system for standardized assembly of multigene constructs. PLoS ONE 2011, 6, e16765. [CrossRef]

51. Engler, C.; Youles, M.; Gruetzner, R.; Ehnert, T.-M.; Werner, S.; Jones, J.; Patron, N.J.; Marillonnet, S. A golden gate modular cloning toolbox for plants. ACS Synth. Biol. 2014, 3, 839-843. [CrossRef]

52. Mehrshahi, P.; Nguyen, G.T.D.T.; Rovira, A.G.; Sayer, A.; Llavero-Pasquina, M.; Sin, M.L.H.; Medcalf, E.J.; Mendoza-Ochoa, G.I.; Scaife, M.A.; Smith, A.G. Development of novel Riboswitches for synthetic biology in the green alga Chlamydomonas. ACS Synth. Biol. 2020, 9, 1406-1417. [CrossRef]

53. Lumbreras, V.; Stevens, D.R.; Purton, S. Efficient foreign gene expression in Chlamydomonas reinhardtii mediated by an endogenous intron. Plant J. 1998, 14, 441-447. [CrossRef]

54. Wichmann, J.; Baier, T.; Wentnagel, E.; Lauersen, K.; Kruse, O. Tailored carbon partitioning for phototrophic production of (E)- $\alpha$-bisabolene from the green microalga Chlamydomonas reinhardtii. Metab. Eng. 2018, 45, 211-222. [CrossRef]

55. Shen, Y.; Liu, Y.; Liu, L.; Liang, C.; Li, Q.Q. Unique features of nuclear mRNA poly (A) signals and alternative polyadenylation in Chlamydomonas reinhardtii. Genetics 2008, 179, 167-176. [CrossRef]

56. Helliwell, K.E.; Scaife, M.A.; Sasso, S.; Araujo, A.P.U.; Purton, S.; Smith, A. Unraveling vitamin B12-responsive gene regulation in algae. Plant Physiol. 2014, 165, 388-397. [CrossRef] [PubMed]

57. Park, J.; Wang, H.; Gargouri, M.; Deshpande, R.R.; Skepper, J.N.; Holguin, F.O.; Juergens, M.T.; Shachar-Hill, Y.; Hicks, L.M.; Gang, D.R. The response of Chlamydomonas reinhardtii to nitrogen deprivation: A systems biology analysis. Plant J. 2015, 81, 611-624. [CrossRef]

58. Purton, S.; Szaub, J.B.; Wannathong, T.; Young, R.; Economou, C.K. Genetic engineering of algal chloroplasts: Progress and prospects. Russ. J. Plant Physiol. 2013, 60, 491-499. [CrossRef]

59. Economou, C.; Wannathong, T.; Szaub, J.; Purton, S. A simple, low-cost method for chloroplast transformation of the green alga Chlamydomonas reinhardtii. Recent Results Cancer Res. 2014, 1132, 401-411. [CrossRef]

60. Ramundo, S.; Rahire, M.; Schaad, O.; Rochaix, J.-D. Repression of essential chloroplast genes reveals new signaling pathways and regulatory feedback loops in Chlamydomonas. Plant Cell 2013, 25, 167-186. [CrossRef] [PubMed]

61. Lauersen, K.J.; Berger, H.; Mussgnug, J.H.; Kruse, O. Efficient recombinant protein production and secretion from nuclear transgenes in Chlamydomonas reinhardtii. J. Biotechnol. 2013, 167, 101-110. [CrossRef]

62. Lauersen, K.J.; Baier, T.; Wichmann, J.; Wördenweber, R.; Mussgnug, J.H.; Hübner, W.; Huser, T.; Kruse, O. Efficient phototrophic production of a high-value sesquiterpenoid from the eukaryotic microalga Chlamydomonas reinhardtii. Metab. Eng. 2016, 38, 331-343. [CrossRef]

63. Zones, J.M.; Blaby, I.K.; Merchant, S.S.; Umen, J.G. High-resolution profiling of a synchronized diurnal transcriptome from Chlamydomonas reinhardtii reveals continuous cell and metabolic differentiation. Plant Cell 2015, 27, 2743-2769. [CrossRef] 